\title{
Three-Dimensional Crane Modelling and Control Using Euler-Lagrange State-Space Approach and Anti-Swing Fuzzy Logic
}

\author{
Andrei Aksjonov (Doctoral student, Tallinn University of Technology), Valery Vodovozov (Professor, Tallinn \\ University of Technology), Eduard Petlenkov (Associate professor, Tallinn University of Technology)
}

\begin{abstract}
The mathematical model of the three-dimensional crane using the Euler-Lagrange approach is derived. A statespace representation of the derived model is proposed and explored in the Simulink ${ }^{\circledR}$ environment and on the laboratory stand. The obtained control design was simulated, analyzed and compared with existing encoder-based system provided by the three-dimensional (3D) Crane manufacturer Inteco ${ }^{\circledR}$. As well, an anti-swing fuzzy logic control has been developed, simulated, and analyzed. Obtained control algorithm is compared with the existing anti-swing proportional-integral controller designed by the 3D crane manufacturer Inteco ${ }^{\circledR}$. 5-degree of freedom (5DOF) control schemes are designed, examined and compared with the various load masses. The topicality of the problem is due to the wide usage of gantry cranes in industry. The solution is proposed for the future research in sensorless and intelligent control of complex motor driven application.
\end{abstract}

Keywords - Control systems; Lagrangian functions; Multidimensional systems; Fuzzy control; Variable speed drives.

\section{INTRODUCTION}

Nowadays, the three-dimensional (3D) cranes also known as overhead gantry cranes are widely used in industry. Particularly, for vessel loading and unloading, to handle the various masses and dimensions, lift containers, etc. They are applied in power industry, automotive lines, ship and airplane building and other heavy manufacturing.

Contemporary cranes are operated mostly manually. This makes the system dangerous because of the numerous accidents and accuracy loss. The acceleration or deceleration of the crane may result in undesirable load swing causing human accidents or damage the crane or the load. To control the swing, the operators need to be experienced in the crane maneuvering. New algorithms used in automated systems may help the operator to control the load swing or can even replace the operator.

Today, the cranes are being built with semi- or fullautomation capability. Many studies have been conducted in order to find the best, cheapest, and optimal solution to control cranes. Researches in the area are intended to improve the accuracy and to minimize the system human interface resulting in the increasing work efficiency provided by the cranes, avoiding accidents, and minimizing dependence on the system operator by involving automation control.

Thanks to diminishing of the human factor risk, the system may achieve precise control and improve load positioning. Thus, the control problem plays an important role in crane system engineering. The main problems still remain in the three-dimensional crane accurate positioning and in the load swing elimination during the motion.

To control the crane speed and positioning, many different controllers have been offered, including the classical proportional-integral (PI), proportional-differential (PD), and proportional-integral-differential (PID) controllers, linearquadratic regulators, model predictive control systems, intelligent fuzzy logic controllers (FLC), and many others. All the controllers have their own advantages and disadvantages.

At present, the intelligent control schemes built on the fuzzy logic are widely distributed in automation control engineering. Mainly, they are the most suitable and beneficial in both multi-input, multi-output (MIMO) and multi-input, singleoutput (MISO) systems. They have approved their success in process control in many areas such as flight, engine, position and process controls, control of vehicle, navigation, load estimation and many others.

Substantially, some interesting ideas were proposed to replace the encoders with the model-based controllers making the control safer, cheaper and more accurate due to the fact that encoders provide and accumulate errors. Furthermore, it is important to design the control logic stable and adaptive to various masses of the load.

The modeling of the control systems is one of the most important parts in control engineering. It helps to understand both physical and theoretical behaviour of the system. Finally, it helps to choose and design the appropriate control algorithm based on the needs. In this context, researchers from different universities around the world have shown their solutions in modeling and building algorithms for three-dimensional crane system described in [1]-[4]. In [2] is also used the Lagrange theory. The Cartesian coordinate robot is described in [5]. As well as Inteco ${ }^{\circledR}$ proposed in [6] original mathematical model derivation. However, none of the researchers were found where the state-space approach is applied to build the control system for the overhead crane.

In [7] and [8] the fuzzy logic was used to design the control system and in [9] the same approach was applied to provide an anti-swing control. Fuzzy logic control for load swing elimination performance in [10] guarantees both accurate positioning and prompt damping of load swing. Moreover, the author provides the 3D Crane system modelling as well.

Authors of [11] presented the separate fuzzy logic controllers for the 3D Crane trolley and hoisting. The anti- 
swing FLC has been also offered and combined with the trolley control. Mathematical modeling of the overhead gantry crane based on the single pendulum system, along with the digital simulation of the fuzzy logic was shown in [12] and [13]. In the latter, the fuzzy logic control has been compared with the Linear Quadratic controller. In [14] another proportional-derivative (PD)-type fuzzy controller has been designed as an anti-swing algorithm. Nevertheless, the results show only the horizontal position of the cart.

In [15] and [16] an adaptive fuzzy controller for 3D Crane was designed. The results were simulated with varying length of flexible-wire of the load as well with different masses. Unfortunately, the results were guided for the only motion between the two points: initial and final. No information has been provided for the behavior of the overhead gantry crane system with constantly varying length of the lift-line and motion between positions. In this case the oscillation of the payload may not be able to dissolve before the next position motion and the oscillation will remain constant or accumulate the amplitude.

Other anti-swing solutions based on fuzzy logic regulator were proposed in [17]-[20]. The last paper also showed the PID regulator simulations for the 2-position displacement. Finally, the author in [21] theoretically described the difference between PID and fuzzy controllers.

The current paper represents the results obtained by the authors in the mathematical modelling using Euler-Lagrange equation and state-space approach. An anti-swing fuzzy logic control design of the 3D Crane is proposed. Different masses are given to the overhead gantry crane laboratory setup while the FLC experiments conducted. The results are analyzed and compared in the similar experimental conditions tested on the existing PI controller provided by the 3D Crane manufacturer Inteco ${ }^{\circledR}$.

The main tasks of the presented work are as follows:

- to derive the mathematical model for the 3D crane

- to simulate the system using Simulink environment and to compare it with the existing encoder-based system provided by the crane manufacturer

- to tune the derived controller as close as possible to the existing one in order to be able to replace the encoder-based system with the model-based unit

- to design a fuzzy logic payload anti-swing 5DOF control for 3D Crane accurate positioning

- to simulate and compare the results for FLC and PI controllers with different payload masses and varying length of the lifted load.

The paper is organized in the following way. First, the 3D Crane experimental setup is described. Second, the model derivation is explained. Then, the major experimental results are discussed and solutions are drawn. Fourth, the anti-swing 5DOF fuzzy control is designed. Next, the anti-swing 5DOF PI and FLC regulators are simulated at various payload masses and changing load lifting-line. Finally, the simulation and experimental results are compared.

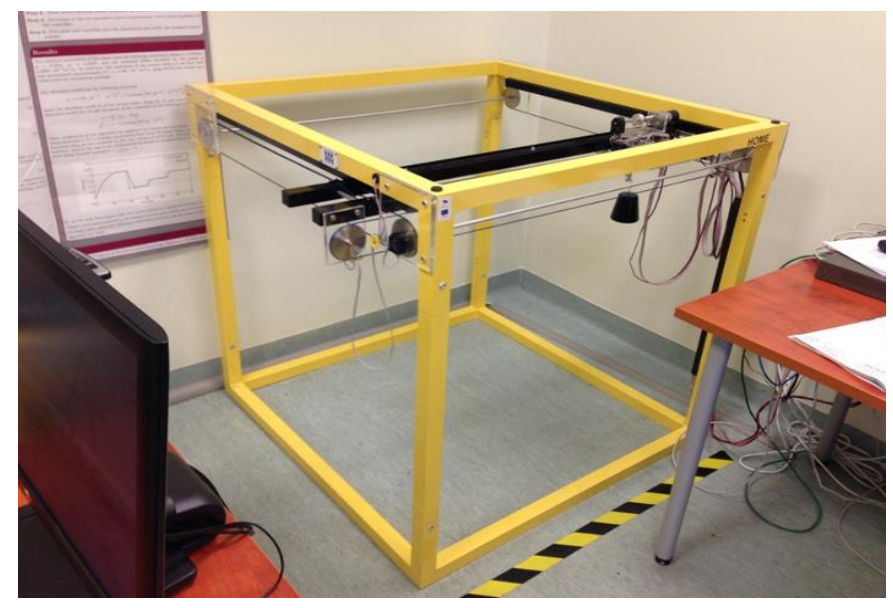

Fig. 1. Laboratory 3D Crane setup.

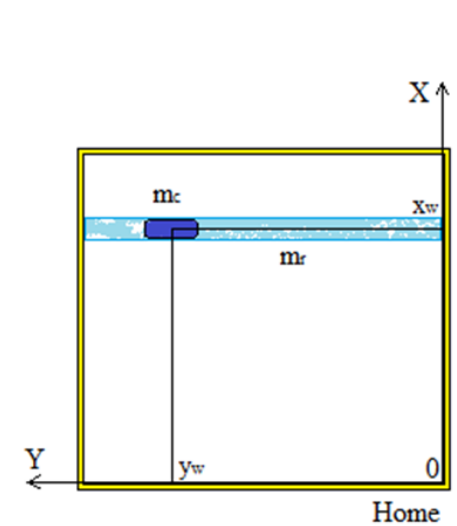

(a)

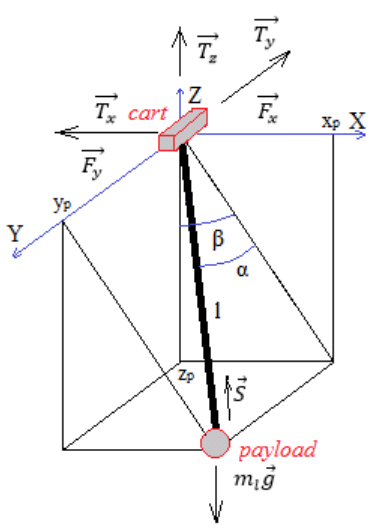

(b)
Fig. 2. 3D Crane: (a) workspace (b) schematic drawing.

TABLE I

3D CRANE DYNAMIC PARAMETERS DESCRIPTION

\begin{tabular}{|c|l|}
\hline Symbol & Description \\
\hline$m_{c}$ & Mass of the cart; $m_{c}=1.16 \mathrm{~kg}$ \\
\hline$m_{l}$ & Mass of the payload; $m_{l}=0.58 \mathrm{~kg}$ \\
\hline$m_{r}$ & Mass of the moving rail; $m_{r}=2.2 \mathrm{~kg}$ \\
\hline$x_{p}$ & $X$-axis position of the payload \\
\hline$y_{p}$ & $Y$-axis position of the payload \\
\hline$z_{p}$ & $Z$-axis position of the payload \\
\hline$g$ & Gravitational constant \\
\hline$F_{x}$ & Force driving the rail with the cart \\
\hline$F_{y}$ & Force driving the cart along the rail \\
\hline$l$ & Length of the lifted load; $l_{(\text {max })}=820 \mathrm{~mm}$ \\
\hline$T_{x}$ & $X$-axis tension force \\
\hline$T_{y}$ & $Y$-axis tension force \\
\hline$S$ & Reaction force acting while lift the load \\
\hline$x_{w}$ & $X$-axis position of the cart in workspace; $x_{w(\max )}=920 \mathrm{~mm}$ \\
\hline$y_{w}$ & $Y$-axis position of the cart in workspace $y_{w(\max )}=920 \mathrm{~mm}$ \\
\hline$\alpha$ & Angle between the lift-line and its projection on $X Y$ \\
\hline$\beta$ & $\begin{array}{l}\text { Angle between the negative direction of the } Z \text {-axis and } \\
\text { projection of the lift-line on } X Y \text { plane }\end{array}$ \\
\hline
\end{tabular}




\section{3D CRANE EXPERIMENTAL SETUP}

The research was conducted on the computer-controlled three-dimensional crane setup 3D Crane from the manufacturer Inteco ${ }^{\circledR}$ (Fig. 1) representing the small copy of an industrial crane [6].

3D Crane control system is suitable for testing complex nonlinear MIMO control algorithms. The crane is driven by three direct current (DC) variable-speed drives. It consists of a payload hanging on a pendulum-like lift-line with a motor mounted on a cart [6]. The crane operation feedback is fulfilled by the Hall-effect switches used as the encoders. Switches measure the positions and the angles of the payload in real-time condition.

The software operates under MS Windows ${ }^{\circledR}$ NT using MATLAB $^{\circledR} /$ Simulink $^{\circledR}$ environment and Real-Time Window Target $^{\mathrm{TM}}$ (RTWT) toolbox package [6]. The control aims to provide the signals to adjust three DC motors, moving along the $X, Y$ and $Z$-axes.

The mounting frame makes a support and a flexible construction of the system. The construction is rigid due to the reason it is fixed on the floor.

The schematic drawing of the 3D Crane and an appropriate workspace are presented in Fig. 2. All the presented dynamic parameters in the Fig. 2 are described in Table I. There are 5 parameters measured by the encoders: $x_{w}, y_{w}, l, \alpha$ and $\beta$. These parameters serve also as the outputs of the control system. Mention that the positions of the cart and the payload are restricted by the crane workspace and lifting-line of the load. The dimensions of the limits are also presented in Table I.

\section{MODEL DESCRIPTION AND SIMULATION}

\section{A. Euler-Lagrange Dynamic Model Derivation}

First, define the mathematical representation of the payload as follows:

$$
\begin{gathered}
x_{w}=x \\
y_{w}=y \\
x_{p}=x+l \cos \alpha \sin \beta \\
y_{p}=y+l \sin \alpha \\
z_{p}=-l \cos \alpha \cos \beta
\end{gathered}
$$

The dynamic representation of the model is derived using the Lagrangian approach. The equation is defined as the difference of kinetic energy of the system $K$ and its potential energy $P$. In our case, $K$ is the kinetic energy of both the crane and its load whereas $P$ defines the potential energy of the load:

$$
L=K-P=\frac{1}{2} m v^{2}-m g l
$$

The speed of the load is defined as:

$$
v^{2}=\dot{x}_{p}^{2}+\dot{y}_{p}^{2}+\dot{z}_{p}^{2}
$$

The Euler-Lagrange formulation of the dynamic system:

$$
\frac{d}{d t}\left(\frac{\partial L}{\partial \dot{q}_{i}}\right)-\frac{\partial L}{\partial q_{i}}=F_{i}
$$

where $i=1,2,3,4,5$

The variable $q$ relates to a generalized coordinate for each output. The final result of the dynamic system for the $3 \mathrm{D}$ Crane is represented by 5 nonlinear second-order differential equations.

\section{B. State-Space Representation}

The dynamic model obtained is highly nonlinear multivariable system. In order to design the control for the $3 \mathrm{D}$ Crane, the state-space representation is used as the best instrument to highly study complicated physical systems. However, before the control design, the system dynamical equations may be subjected to the approximations. Assuming the small deviation of the payload along the $z$-axis executed by the fast controller, the dynamic model can be simplified as follows:

$$
\begin{aligned}
& \sin \alpha \approx \alpha \\
& \cos \alpha \approx 1
\end{aligned}
$$

The input forces for the 3D Crane are stated as the following vector:

$$
u=\left[u_{x} u_{y} u_{z}\right]^{T}
$$

The state-space operational point is stated as follows:

$$
x^{0}=\left[x^{0} \dot{x}^{0} y^{0} \dot{y}^{0} \beta^{0} \dot{\beta}^{0} \alpha^{0} \dot{\alpha}^{0} l^{0} \dot{l}^{0}\right]^{T}
$$

The $A, B, C$ and $D$ matrices are calculated taking into account the steady-state operation point $x^{0}=0$ and input forces vector $u=0$. These approximations are adequate due to the rule where all the derivatives of the state variables, measured variables, and input forces must be equal to zero when the expected steady-state position of the cart is obtained.

Finally, the state equations are given by

$$
\begin{aligned}
& \dot{x}_{1}=\dot{x}_{w}=x_{2} \\
& \dot{x}_{2}=\ddot{x}_{w}=\frac{1}{m_{c}+m_{r}+m_{l}} u_{x}+\frac{m_{l} g}{m_{c}+m_{r}+m_{l}} \beta \\
& \dot{x}_{3}=\dot{y}_{w}=x_{4} \\
& \dot{x}_{4}=\ddot{y}_{w}=\frac{1}{m_{c}+m_{l}} u_{y}+\frac{m_{l} g}{m_{c}+m_{l}} \alpha \\
& \dot{x}_{5}=\dot{\beta}=x_{6} \\
& \dot{x}_{6}=\ddot{\beta}=-\frac{1}{l\left(m_{c}+m_{r}+m_{l}\right)} u_{x}-\frac{g\left(m_{c}+m_{r}+2 m_{l}\right)}{l\left(m_{c}+m_{r}+m_{l}\right)} \beta \\
& \dot{x}_{7}=\dot{\alpha}=x_{8} \\
& \dot{x}_{8}=\ddot{\alpha}=-\frac{1}{l\left(m_{c}+m_{l}\right)} u_{y}-\frac{g\left(m_{c}+2 m_{l}\right)}{l\left(m_{c}+m_{l}\right)} \alpha \\
& \dot{x}_{9}=\dot{z}_{w}=x_{10} \\
& \dot{x}_{10}=\ddot{z}=\frac{1}{2 m_{l}} u_{z}
\end{aligned}
$$


All the differential equations for the state-space $A, B, C$ and $D$ matrix elements have been calculated using the MATLAB ${ }^{\circledR}$ operation diff.

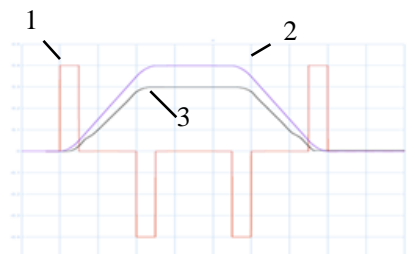

(a)

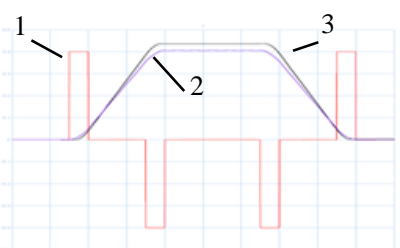

(b)

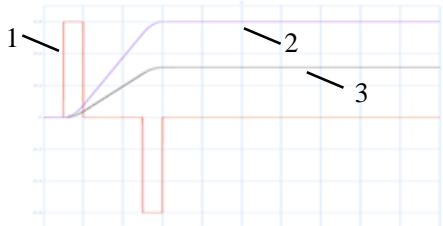

(c)

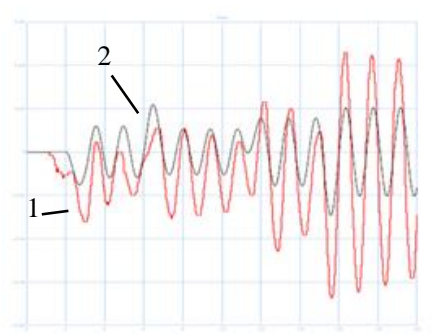

(d)

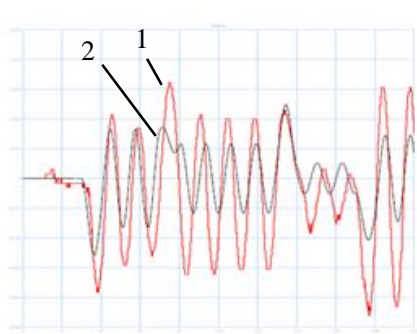

(e)
Fig. 3. The 3D Crane state-space representation and Inteco ${ }^{\mathbb{B}}$ model simulation results: (a) $X$-axis output, (b) $Y$-axis output, (c) $Z$-axis output, (d) $\beta$ angle of payload output, (e) $\alpha$ angle of payload output. In (a) - (c): 1 - input force, 2 state-space representation and 3 - Inteco ${ }^{\circledR}$ model. In (d) and (e): 1 - the output of Inteco ${ }^{\circledR}$ model and 2 - the state-space output.

\section{Simulation}

Both the designed state-space and Inteco ${ }^{\circledR}$ models were located in the same Simulink ${ }^{\circledR}$ workspace window and the same input signals were applied to both models. Three traces are added to view the input, the Inteco ${ }^{\circledR}$ output of the model and the output of the state-space representation. Therewith, the additional traces are placed to observe and study the outputs of the payload angles what cause the payload oscillation problem.

As the experimental results did not completely match the state-space representation output, the correction gain has been found to scale axes positions for the state-space approach. The output position respond is easy to tune by simple dividing the output steady-state value to the input force value. The gain $=$ $\left[\begin{array}{lll}1.33 & 0.8 & 0.5\end{array}\right]^{T}$ was chosen.

The simulation results are presented in Fig. 3. The positions along the $X, Y$ and $Z$-axes were designed to follow the cart and the payload lift-line according to the input force. The control logic has been also tuned according to the design.

Analysis shows that there exist some error between the encoder outputs and the state-space representation of the model derived using the Euler-Lagrange approach. Particularly, the amplitudes are not the same comparing to the 3D Crane model and state-space representation, though the frequencies of the oscillation for both $\alpha$ and $\beta$ angels are very close to each other.

\section{FLC}

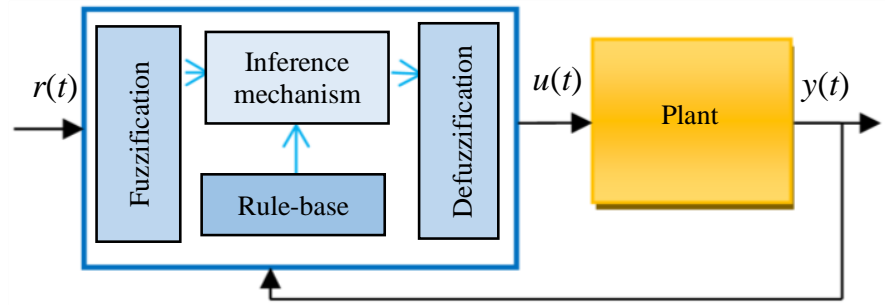

Fig. 4. Fuzzy logic controller block diagram: FLC - fuzzy logic controller, $r(t)-$ reference input, $u(t)$ - process inputs, $y(t)$ - process.
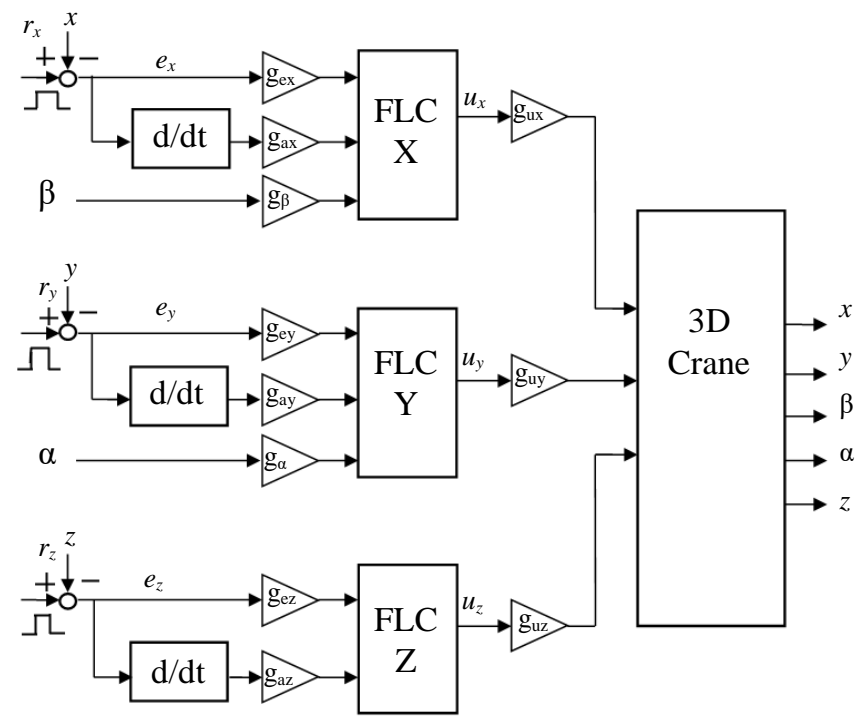

Fig. 5. 3D Crane fuzzy logic control system: $r_{x}, r_{y}$ and $r_{z}-$ reference $X, Y$ and $Z$-axes positions; $x, y$ and $z$-real $X, Y$ and $Z$-axes positions, $\beta-X$-axis angle; $\alpha-Y$-axis angle; $e_{x}, e_{y}$ and $e_{z}-X, Y$ and $Z$ position errors; $a_{x}, a_{y}$ and $a_{z}$ change in $X, Y$ and $Z$ position errors; $u_{x}, u_{y}$ and $u_{z}-X, Y$ and $Z$ process inputs; $g$ - scaling gain for appropriate term; FLC X, FLC Y and FLC Z $-X, Y$ and $Z$ fuzzy logic controllers.

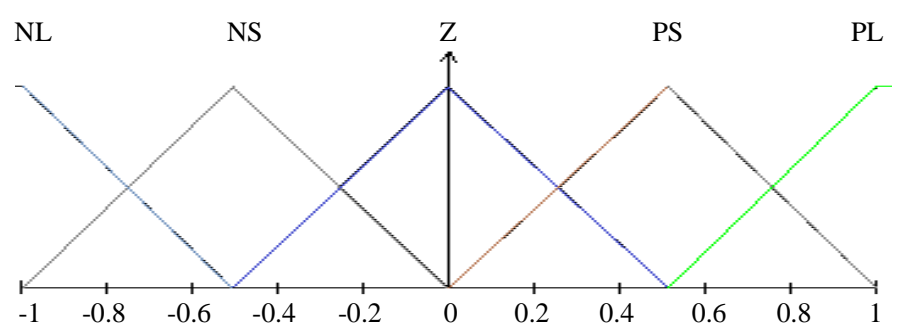

(a)

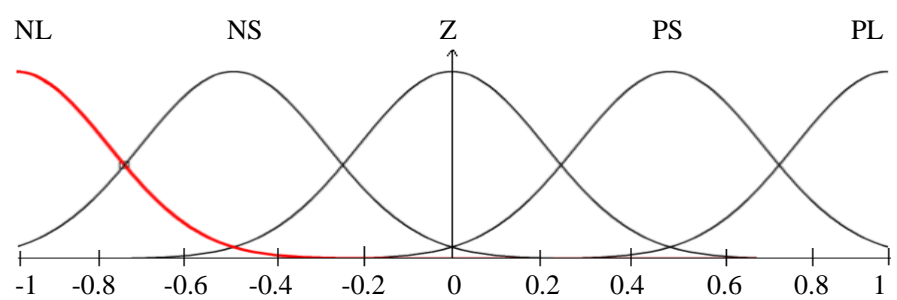

(b)

Fig. 6. Membership function plot for the fuzzy logic controller inputs: NS negative small, NL - negative large, $\mathrm{Z}$ - zero, PS - positive small, PL positive large: (a) input triangular membership function, (b) output Gaussian membership function. 
In general, there can be several reasons explaining, why the encoders and the state-space representation do not match each other in amplitude.

One of the reasons is that many dynamics such as viscose friction coefficient, motors and encoder dynamics have been neglected while modeling the system. Moreover, the disturbances that present in real-world environment are not considered. Also, some other components may influence the system.

Next reason is that the 3D Crane frame can be tilted. Finally not trivial, the encoder errors may appear. Of course, many other features can trouble the correct dynamics.

\section{FUZZY LOGIC CONTROLLER}

A block diagram of fuzzy logic controller is presented in Fig. 4. The fuzzy system is static nonlinear mapping between inputs and outputs [22].

The inputs and outputs of the system are "crisp" meaning that they are real number. The fuzzification block converts the crisp inputs to fuzzy sets. The inference mechanism uses the fuzzy rules in the rule-base to produce fuzzy conclusion. The defuzzification block converts these fuzzy conclusions into crisp outputs [22].

\section{A. Controller Design}

The MATLAB ${ }^{\circledR}$ Fuzzy logic Toolbox is used to design the FLC. First of all, it is important to define the crisp inputs. As it has been mentioned before, the system has three input forces to drive the crane along the $X, Y$ and $Z$-axes. There are five output parameters: $x, y, z, \alpha$ and $\beta$.

According to the scheme in Fig. 4 the outputs of the process are used as the controller inputs. We will therefore have MIMO system with 8 inputs and 3 outputs. For simplicity, the regulation of the $X, Y$ and $Z$-axes will be divided separately onto MISO system.

It is important to note, that only one MIMO controller for all the axes may be designed. At that, the system becomes more complex without any performance improvement.

To develop the anti-swing regulator, the FLC also needs the feedback from the angles $\alpha$ and $\beta$ oscillation. $X$ and $Y$-axes therefore require three inputs and one driving force output for the 3D Crane motors.

As soon as all the inputs and outputs are defined, the control system can be specified. The block diagram of the designed FLC is presented in Fig. 5.

The next step is to plot the membership functions of the controller. The membership function quantifies the meaning of the inputs and outputs. It can be seen in Fig. 6 that for the crisp inputs the triangular membership function is used, whereas the Gaussian membership functions are used for the outputs. The universes of discourse $L$ for inputs and outputs are normalized on the range $[-1,1]$. The system is thus tuned via the scaling universes of discourse with the gains obtained in experiments.

Finally, the design is concluded with the fuzzy linguistic rules. Here it is important to imagine the behavior of the 3D Crane system. Despite that the rules are usually presented in the tables, only few linguistic sentences are presented here, because the 3D Crane system requires more complex logic rules with 3 inputs and 1 output.

In total, there are 125 modus ponens (If-Then) form rules for both $X$ and $Y$-axes. For $Z$-axis there are 25 modus ponens form rules due to excluded angle oscillation output. The examples of the linguistic rules are as follows:

- If error is NL and change-in-error NS and angle PS Then force is NL

- If error is $\mathrm{Z}$ and change-in-error $\mathrm{Z}$ and angle PS Then force is PS

- If error is NS and change-in-error ZE and angle PL Then force is PS

The logic is as follows: if the motion force is very high in positive direction and the angle deviation is very large in negative direction, then apply force in opposite direction.

Precise explanation of how do the decisions in membership functions are made is described in [21] and [22].

\section{B. Tuning the FLC via Scaling Universes of Discourse}

In order to scale the universes of discourse, the classical control engineering method is used. The tuning gains are added to all the inputs and outputs of the fuzzy logic controller. However, it is important to remember that tuning the scaling gains is not the only solution for fuzzy logic controller. The system can be also tuned via membership function shapes, positioning and number and type of rules [22].

As shown in the control logic system (Fig. 5), the scaling gains $g_{e x}, g_{e y}, g_{e z}, g_{a x}, g_{a y}$ and $g_{a z}$ are added to both the proportional and the derivative terms of each of the controlled axis $X, Y$ and $Z$. The gains $g_{u x}, g_{u y}$ and $g_{u z}$ are also placed between the FLC and the 3D Crane. Further as we are considering the information about $\alpha$ and $\beta$ angles of oscillation as another crisp input and their universes of discourse are not known, the gains $g_{\alpha}$ and $g_{\beta}$ are also introduced.

All the mentioned gains are found during the experiment simulation. For the accurate positioning the standard solution was used: decrease the change-in-error term gain and barely increase the error loop gain. On the whole, the scaling gains are chosen as follows: $g_{e x}=2, g_{e y}=2, g_{e z}=0.1, g_{a x}=0.002$, $g_{a y}=0.04, g_{a z}=5, g_{u x}=2, g_{u y}=5, g_{u z}=40, g_{\alpha}=2$ and $g_{\beta}=2$.

\section{EXPERIMENTATION RESULTS}

The simulation positioning results for various payload masses of the PI anti-swing 5DOF control designed by the 3D Crane manufacturer Inteco $^{\circledR}$ are provided in Fig. 7 - Fig. 9. The payload oscillation results for both $\alpha$ and $\beta$ angles are shown in Fig. 10 and Fig. 11.

The simulation positioning results for various payload masses of the anti-swing 5DOF fuzzy logic control designed in this paper are provided in Fig. 12 - Fig. 14. The payload oscillation results for both $\alpha$ and $\beta$ angles are shown in Fig. 15 and Fig. 16.

For the fare comparison the same input forces have been given to the $X, Y$ and $Z$-axes during the simulations. In addition, before every simulation the object have been placed 
to "Home" position, where all the axes locations are equal to 0 as well as the encoders are reset to initial values.

Various payload masses have been given to the 3D Crane in order to observe the differences and adaptation of the controllers to the different conditions. Payload masses as well as the reference position lines colors are shown in the box for every plot in Fig. 7 - Fig. 16.

Although, the respond of the $Z$-axis for both anti-swing PI and fuzzy logic controllers are quite similar for every load mass (Figs. 9 and 14), the controllers have significant differences. $Z$-axis control is necessary to study the impact on the regulators positioning and anti-swing control with changing payload lifting-line.

It can be clearly seen from Figs. 7 and 8 that the positioning in $X$ and $Y$-axes for PI controller is very accurate. However, it can be viewed the endpoint disturbance. The behavior is due to controller reaction on the payload oscillation angle. On one hand, PI controller has fast respond to positioning. On another hand, the oscillation of the load may reach up to 10 degrees, what makes the cart and the rail bounce near the reference position in order to eliminate the swing.

The oscillation angles $\alpha$ (Fig. 11) and $\beta$ (Fig. 10) for antiswing PI regulator are very large. Not to mention, the angles cannot be stabilized before the next motion. The payload remains with the slight oscillation before another pulse.

Fuzzy logic controller, on the contrary, is not showing the precise positioning, what can be viewed in Fig. 12 and Fig. 13. The respond is quite fast, though it becomes slower getting close to the reference point. The reason behind it may be the "fuzzy" rule-based decision: when positioning is around the reference point, the force applied starts to diminish slowly. The positioning error in this case is approximately $0.025 \mathrm{~m}$ for both $X$ and $Y$-axes, what can cause a problem for bigger dimension system.

Nevertheless, the designed anti-swing fuzzy logic controller assimilates the payload mass change very well. Moreover, the elimination of the oscillation of the payload (Figs. 15 and 16) is much better comparing to the PI regulator what appears to be the goal of the designed control algorithm.

Notwithstanding the positioning error, both $\alpha$ (Fig. 16) and $\beta$ (Fig. 15) angles can be stabilized around 0 degree before the next motion. Also the swing index does not exceed 6 degrees for $\beta$ and 8 degrees for $\alpha$ while in PI regulator both angles are higher. Again, neither the influence of the various payload masses nor the lifting-line changes have been descried to impact on the payload swing elimination.

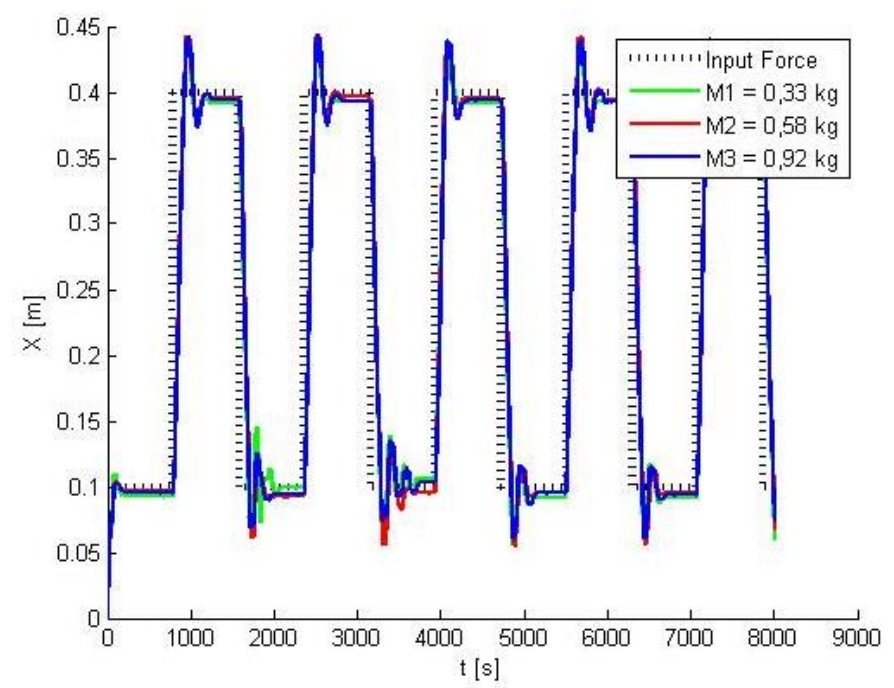

Fig. 7. Anti-swing 5DOF PI regulator $X$-axis position simulation results.

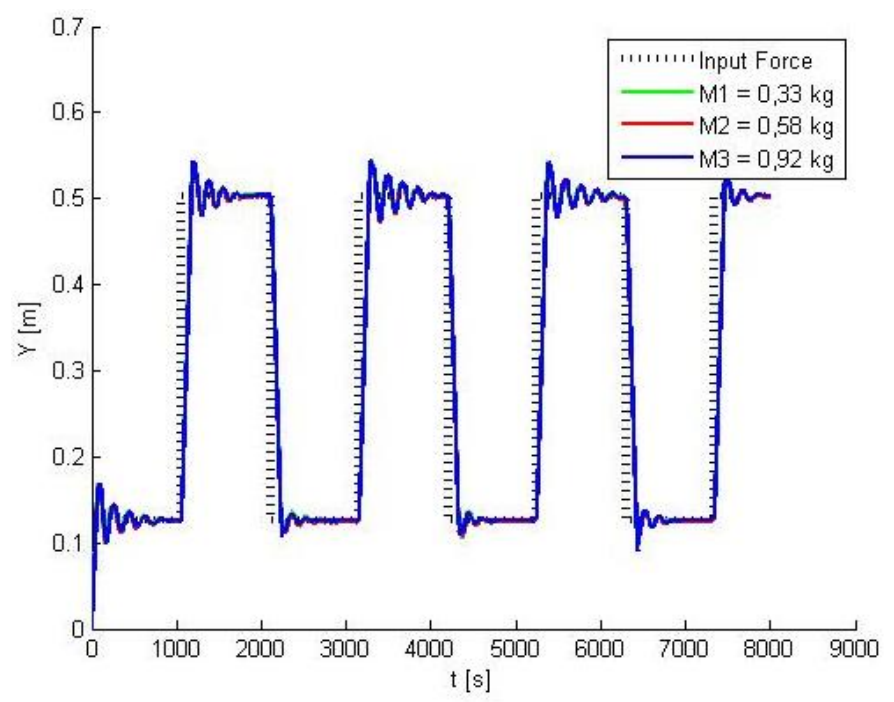

Fig. 8. Anti-swing 5DOF PI regulator $Y$-axis position simulation results.

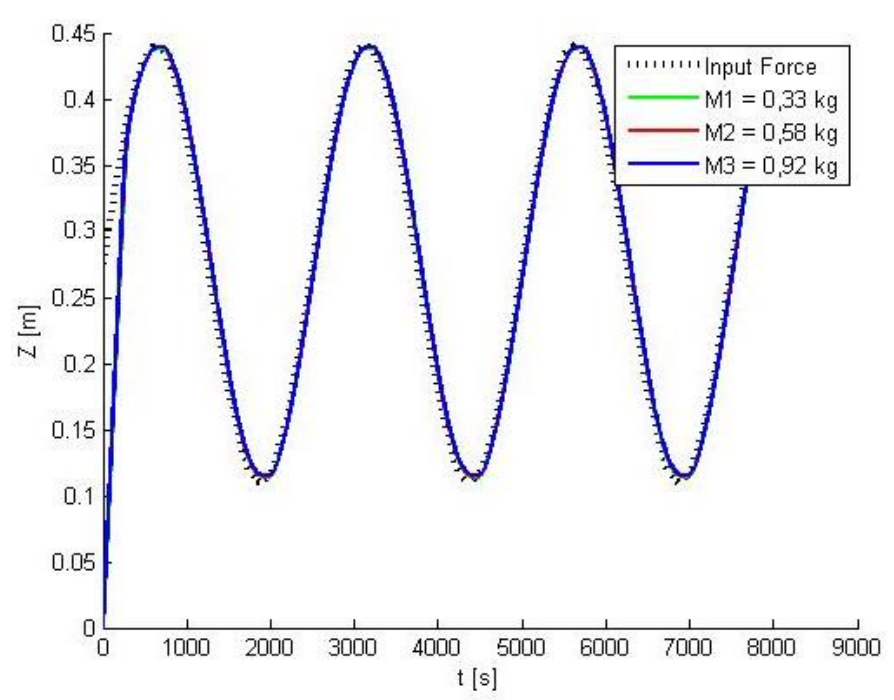

Fig. 9. Anti-swing 5DOF PI regulator $Z$-axis position simulation results. 


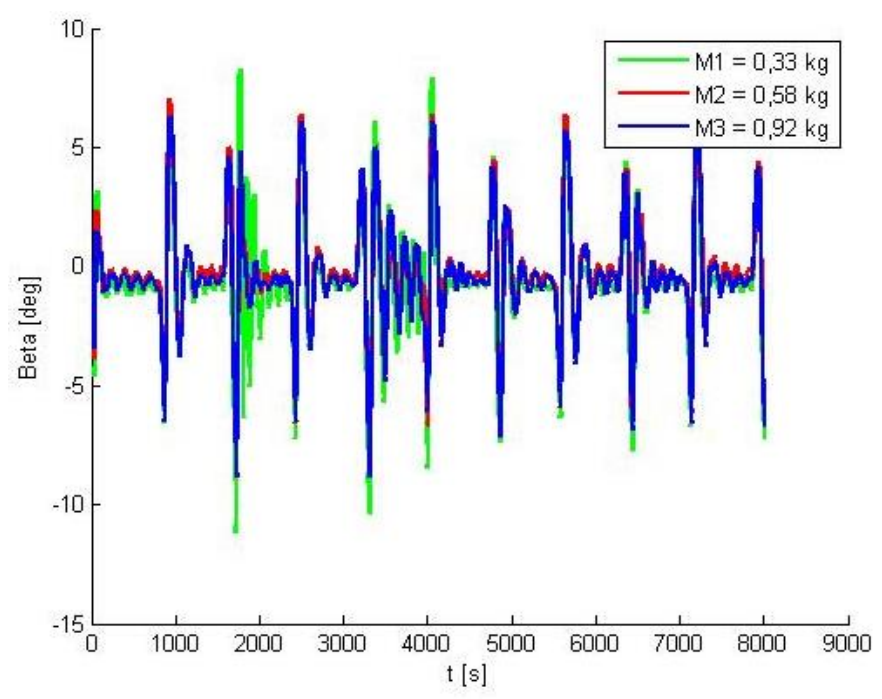

Fig. 10. Anti-swing 5DOF PI regulator $\beta$ angle of the payload oscillation simulation results.

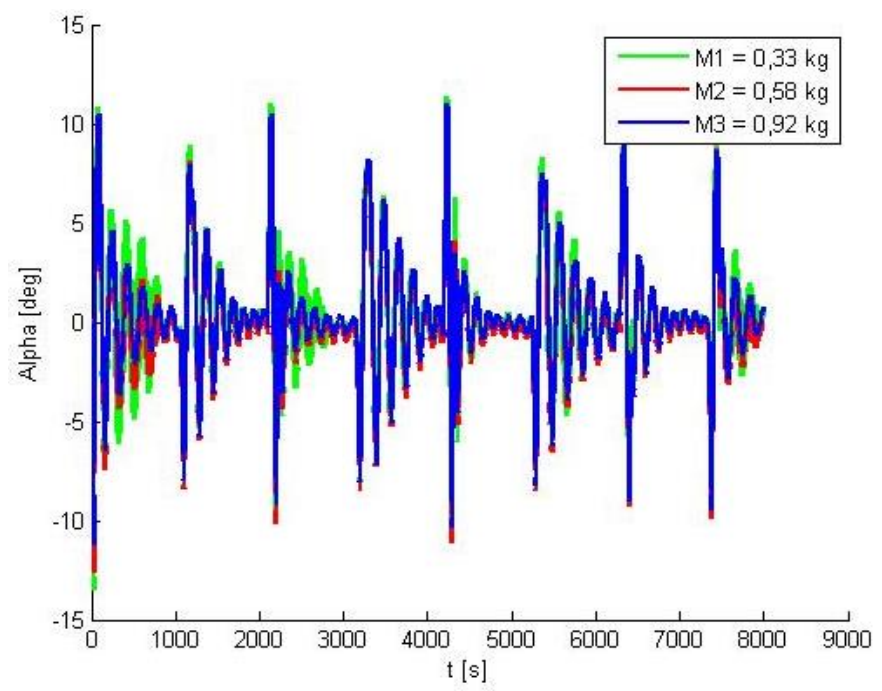

Fig. 11. Anti-swing 5DOF PI regulator $\alpha$ angle of the payload oscillation simulation results.

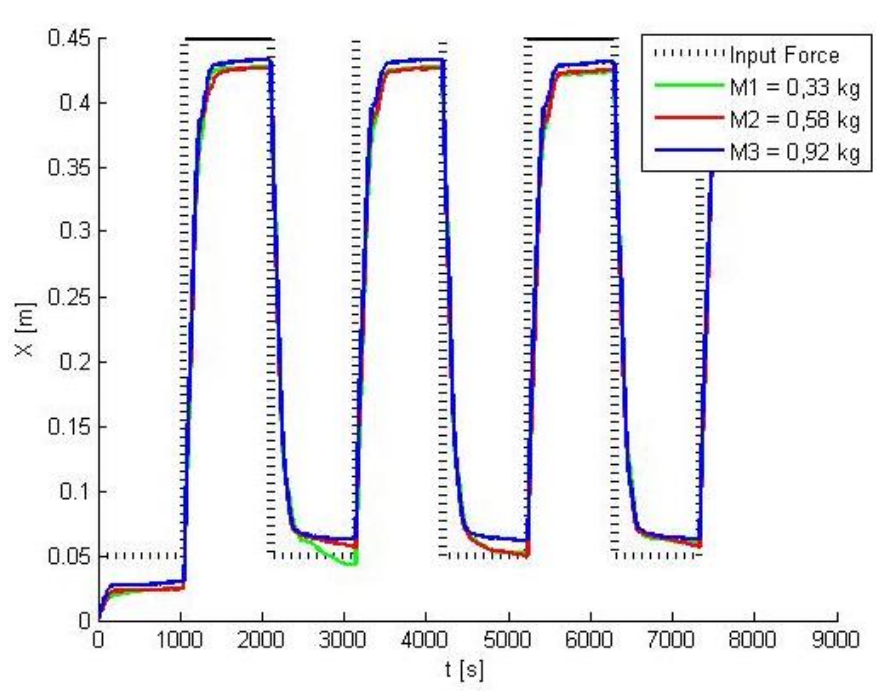

Fig. 12. Anti-swing 5DOF fuzzy logic regulator $X$-axis position simulation results.

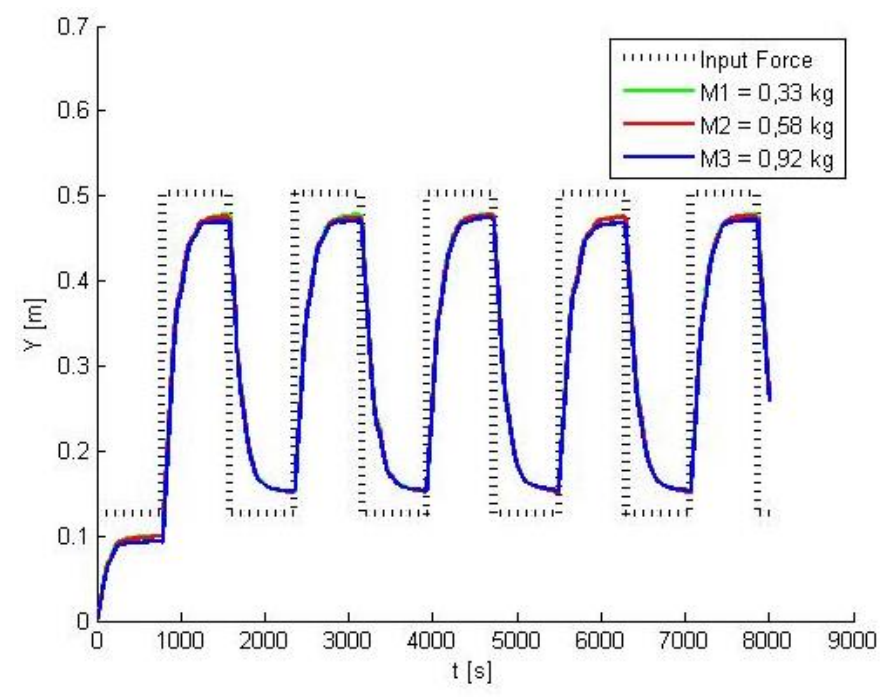

Fig. 13. Anti-swing 5DOF fuzzy logic regulator $Y$-axis position simulation results.

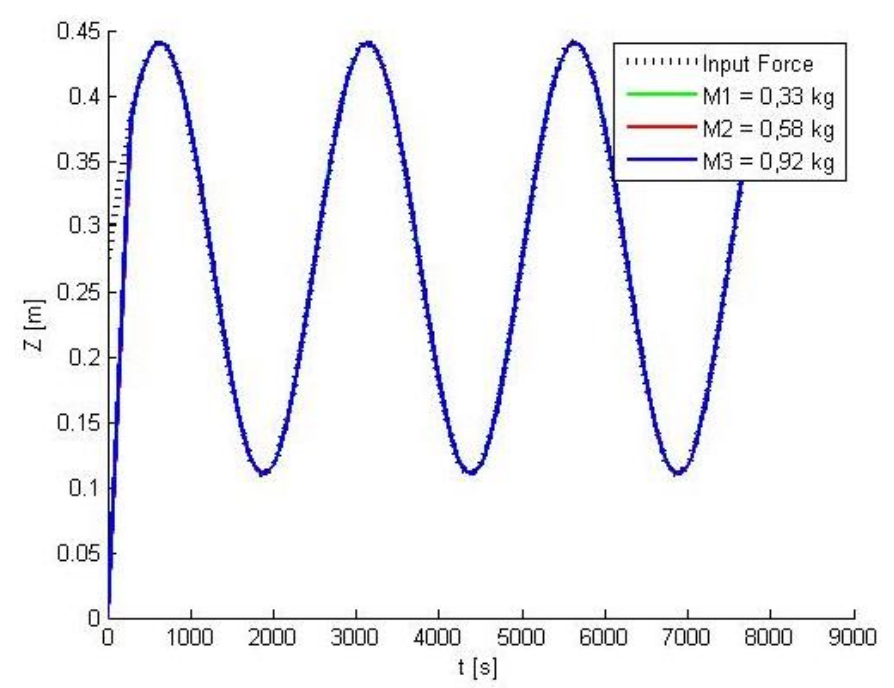

Fig. 14. Anti-swing 5DOF fuzzy logic regulator $Z$-axis position simulation results.

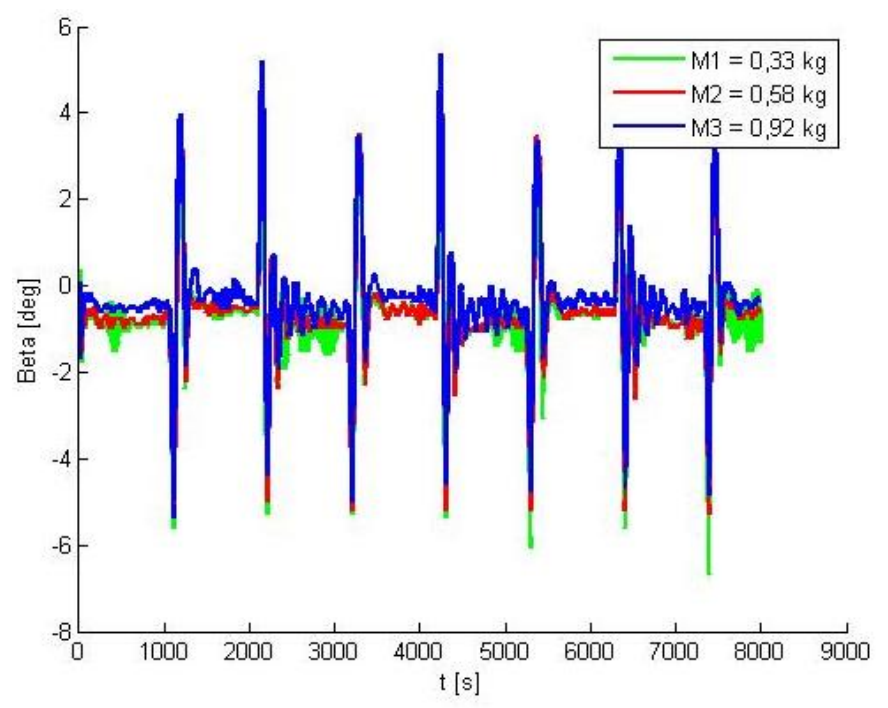

Fig. 15. Anti-swing 5DOF fuzzy logic regulator $\beta$ angle of the payload oscillation simulation results. 


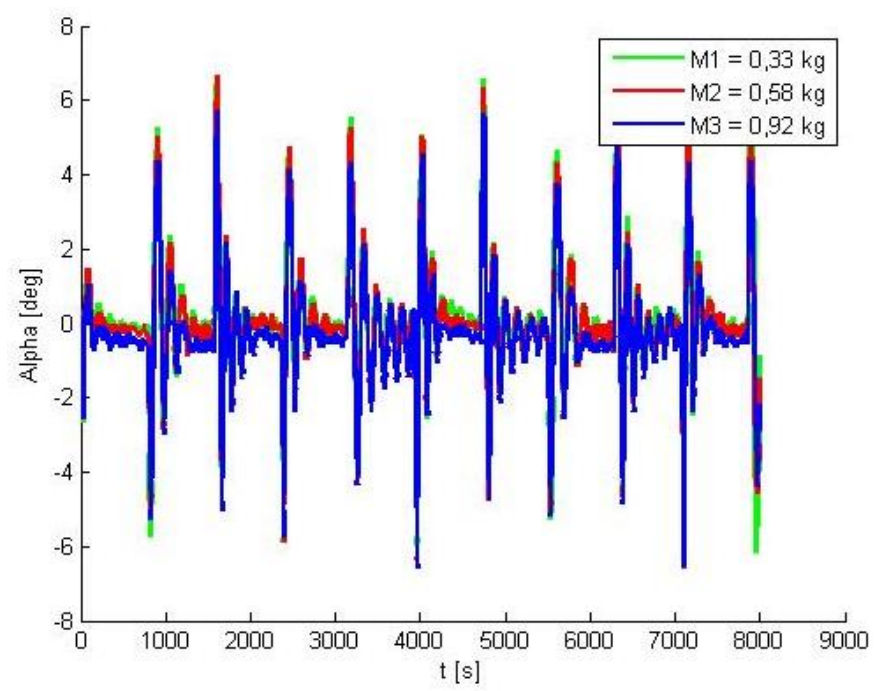

Fig. 16. Anti-swing 5DOF fuzzy logic regulator $\alpha$ angle of the payload oscillation simulation results.

\section{CONCLUSION}

The results obtained from the mathematical model of the three-dimensional crane show that the crane motion and the positioning can be tuned with the correction gain for every axis. The state-space model behaves as expected and the axes move according to the value of input force. As well, the study of the system errors shows that the angles of the payload still provide some mismatch in amplitude, though the oscillation frequency for both $\alpha$ and $\beta$ angels nevertheless is very similar. The system developed can be recommended for the accurate and safe 3D gantry crane control in industrial applications.

Comparison of the anti-swing fuzzy logic controller for the three-dimensional crane and the fuzzy logic five degree of freedom controller show that the PI regulator is easier to design comparing to fuzzy logic regulator. Though the positioning in 5DOF fuzzy logic controller has an error, it is still more stable and accurate comparing to PI controller. Furthermore, the anti-swing task is well carried by FLC comparing to anti-swing PI controller, what is in the current case the goal of the research.

The presented study opens the directions to the future research that may cover the finding of algorithms to scale the fuzzy logic controller membership function universes of discourse for several experimental objects. Different types of cranes such as three-dimensional and tower cranes can be explored using the offered algorithms. Self-scaling intelligent fuzzy logic controller for the multidimensional motor driven applications can be proposed in this way.

\section{REFERENCES}

[1] R. M. T. Raja Ismail, M. A. Ahmad, M. S. Ramli and F. R. M. Rashidi, "Nonlinear Dynamic Modelling and Analysis of a 3-D Overhead Gantry Crane System with System Payload Variation," in 3rd European Symp. on Computer Modeling and Simulation, Athens, Greece, 2009, pp. 350 354. http://dx.doi.org/10.1109/EMS.2009.71

[2] H. Chen, B. Gao and X. Zhang, "Dynamical Modelling and Nonlinear Control of a 3D Crane," in 2005 International Conference on Control and Automation (ICCA2005), Budapest, Hungary, 2005, pp. 1085-1090. http://dx.doi.org/10.1109/ICCA.2005.1528283
[3] M. Z. Mohd Tumari, M. S. Saealal, M. R. Ghazali and Y. Abdul Wahab, "H controller with graphical LMI region profile for Gantry Crane System," in The 18 ${ }^{\text {th }}$ Int. Symp. on Artificial Life and Robotics (AROB 18th '13), Daejeon, Korea, 2013, pp. 346-351. http://umpir.ump.edu.my/3439/

[4] H.-H. Lee, "Modeling and Control of a Three-Dimensional Overhead Crane," Journal of Dynamic Systems, Measurement, and Control, vol. 120 , pp. 471-476, 1998. http://dx.doi.org/10.1115/1.2801488

[5] M. Callegari, F. Cannella, S. Monti, C. Santolini, "Dynamic Models for the Re-Engineering of a High-Speed Cartesian Robot," in 2001 IEEE/ASME Int. Conf. on Advanced Intelligent Mechatronics, Como, Italy, 2001, pp. 560-565. http://dx.doi.org/10.1109/aim.2001.936525

[6] Inteco Limited 3D Crane User's Manual: MATLAB R2009a/b, $\mathrm{R} 2010 \mathrm{a} / \mathrm{b}, \quad \mathrm{R} 2011 \mathrm{a} / \mathrm{b} \quad \mathrm{PCI} \quad$ version, $\quad$ Available: http://www.inteco.com.pl/products/3d-crane/ [Accessed: Feb. 20, 2015].

[7] P. Petrehus, Zs. Lendek and P. Raica, "Fuzzy modeling and design for a 3D Crane," in $3^{\text {rd }}$ IFAC Int. Conf. on Intelligent Control and Automation Science, Chengdu, China, 2013, pp. 479-484. http://dx.doi.org/10.3182/20130902-3-cn-3020.00058

[8] D. M. Trajković, D. S. Antić, S. S. Nikolić, S. Lj. Perić and M. B. Milovanović, "Fuzzy Logic-Based Control of Three-Dimensional Crane System," Automatic Control and Robotics. vol. 12, no. 1, pp. 3142, 2013

[9] Z. Jovanović, A. Perić, S Nikolić, M. Milojković and M. Milosević, "Anti-Swing Fuzzy Controller Applied in a 3D Crane System," ETASR Engineering, Technology \& Applied Science Research, vol. 2, no. 2, pp. 196- 200, 2012.

[10] H.-H. Lee and S. Cho, "A New Fuzzy-Logic Anti-Swing Control for Industrial Three-Dimensional Overhead Cranes," in 2001 IEEE Int. Conf. on Robotics \& Automation, Seoul, Korea, 2001, pp. 2956- 2961. http://dx.doi.org/10.1109/ROBOT.2001.933070

[11] M. B. Trabia, J. M. Renno and K. A. F. Noustafa, "A General AntiSwing Fuzzy Controller for an Overhead Crane with Hoisting," in 2006 IEEE Int. Conf. on Fuzzy Systems, Vancouver, BC, Canada, 2006, pp. 627- 634. http://dx.doi.org/10.1109/FUZZY.2006.1681777

[12] Z. Nowacki, D. Owczarz and P. Wozniak, "On the Robustness of Fuzzy Control of an Overhead Crane," in Proceedings of the IEEE International Symposium on Industrial Electronics, ISIE '96. Warsaw, Poland, 1996, pp. 433- 437. http://dx.doi.org/10.1109/isie.1996.548527

[13] T. Popadic, F. Kolonic and A. Poljugan, "A Fuzzy Control Scheme for the Gantry Crane Position and Load Swing Control," Available: https://bib.irb.hr/datoteka/248540.MIPRO_FuzzySPG_V4.pdf [Accessed: Oct. 18, 2015]

[14] M. A. Ahmad, M. S. Ramil, R. M. T. Raja Ismail, A. N. K. Nasir and M. A. Zawawi, "The investigation of PD-type Fuzzy Logic with different polarities input shaping for anti-sway control of a gantry crane system," in 2009 Conf. on Innovative Technologies in Intelligent System and Industrial Applications (CITISIA 2009), Monash, Malaysia, 2009, pp. 452- 457. http://dx.doi.org/10.1109/CITISIA.2009.5224166

[15] C.-Y. Chang, "Adaptive Fuzzy Controller of the Overhead Crane With Nonlinear Disturbance," IEEE Trans. on Ind. Informat., vol. 3, no. 2, pp. 164- 172, 2007. http://dx.doi.org/10.1109/TII.2007.898433

[16] J. Smoczek and J. Szpytko, "Fuzzy Logic-based Adaptive Control System Prototypying for Laboratory Scaled Overhead Crane," in $18^{\text {th }}$ Int. Conf. on Methods and Models in Automation and Robotics (MMAR), Miedzyzdroje, Poland, 2013, pp. 92- 97. http://dx. doi. org/10.1109/mmar. 2013.6669887

[17] Y. Zhao and H. Gao, "Fuzzy-Model-Based Control of an Overhead Crane With Input Delay and Actuator Saturation," IEEE Trans. on Fuzzy Systems, vol. 20, no. 1, pp. 181- 186, 2012. http://dx.doi.org/10.1109/TFUZZ.2011.2164083

[18] S. Carmeli and M. Mauri, "HIL test bench to test anti-swing fuzzy control of an overhead crane," in 2013 IEEE Int. Conf. on Mechatronics (ICM), Vicenza, Italy, 2013, pp. 754- 760 http://dx.doi.org/10.1109/ICMECH.2013.6519136

[19] C.-Y. Chang, K.-C. Hsu, K.-H. Chiang and G.-E. Huang, "An Enhanced Adaptive Sliding Mode Fuzzy Control for positioning and Anti-Swing Control of the Overhead Crane System," 2006 IEEE Int. Conf. on System, Man, and Cybernetics, Taipei, Taiwan, 2006, pp. 992- 997. http://dx.doi.org/10.1109/ICSMC.2006.384529

[20] L. Wang, H. Zhang and Z. Kong, "Anti-swing Control of Overhead Crane Based on Double Fuzzy Controller," in $27^{\text {th }}$ Chinese Control and 
Design Conf. (CCDC), Qingdao, China, 2015, pp. 981- 986. http://dx.doi.org/10.1109/ccdc.2015.7162061

[21] J. Goljevac, "Comparison Between PID and Fuzzy Control," Ecole Polytechnique Fédérale de Lausanne, Département d'Informatique, Laboratoire de Microinformatique, Internal Report R93.36I.

[22] K. M. Passino and S. Yurkovich, Fuzzy Control. California, USA: Addison Wesley Longman, Inc., 1998.

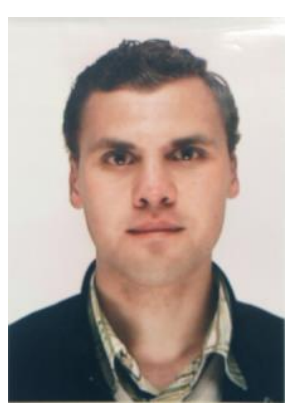

Andrei Aksjonov received B. Sc. and M. Sc. in electrical engineering and electrical drives and power electronics with automation specialization from Tallinn University of Technology (TUT) in 2013 and 2015, respectively. His current research topic is "Design and Implementation of Intellectual Fuzzy Regulators and Observers for Multidimensional Motor Driven Applications".

Postal address: Ehitajate Tee 5, 19086, Tallinn, Estonia.

E-mail: andrei.aksjonov@outlook.com

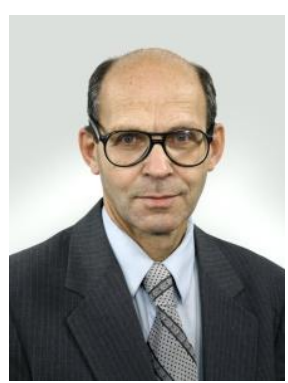

Valery Vodovozov received his Candidate of Science degree and Associate Professor and Senior Researcher academic titles in Electrical Engineering from St. Petersburg Electrotechnical University, Russia. He is a professor of Tallinn University of Technology, a member of IEEE, International Institute of Informatics and Systemics (IIIS), and Global Research Alliance of Texas Institute of Science (TxIS GRA).

Postal address: Ehitajate Tee 5, 19086, Tallinn, Estonia.

E-mail: valery.vodovozov@ttu.ee

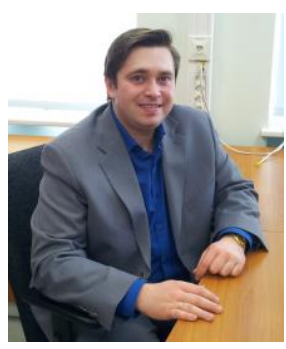

Eduard Petlenkov received his B. Sc., M. Sc. and $\mathrm{PhD}$ degrees in computer and systems engineering from Tallinn University of Technology. He is an Associate Professor in the Department of Computer Control at Tallinn University of Technology. His main research interests lie in the domain of nonlinear control, system analysis and computational intelligence.

Postal address: Ehitajate Tee 5, 19086, Tallinn,

Estonia.

E-mail: eduard.petlenkov@ttu.ee 